\title{
LA INFRAESTRUCTURA COMO ESTRATEGIA PARA LA CONSERVACIÓN DEL TERRITORIO

\author{
La obra de Kongjian Yu
}

\author{
INFRASTRUCTURE AS A STRATEGY FOR \\ LAND CONSERVATION \\ Kongjian Yu's Work
}

\author{
INGRID OLÓRTEGUI \\ Blekinge Institute of Technology \\ Universidad Peruana de Ciencias Aplicadas \\ orcid.org/0000-0002-2088-876X
}

El presente artículo explicará las estrategias empleadas por el arquitecto chino Kongjian $\mathrm{Yu}$ y su equipo Turenscape, con el fin de analizar y entender al territorio como punto de partida para el desarrollo de sus propuestas paisajistas, las cuales desafían la tendencia del uso masivo del concreto en China. El regreso a las raíces de la cultura, la naturaleza y el entendimiento del territorio para plantear proyectos que manejen de forma orgánica la variabilidad de la naturaleza, especialmente en su relación con el agua, será relacionado con el incipiente desarrollo de la infraestructura natural en el ámbito peruano. La experiencia de Yu logra vincular la llamada infraestructura ecológica con el desarrollo urbano, cuyo símil en el Perú sería la infraestructura natural, que se concibe como el desarrollo del espacio natural asociado al origen de las fuentes de agua en áreas rurales, prevaleciendo aún la implementación de la infraestructura gris en las ciudades.

infraestructura natural, infraestructura verde, seguridad hídrica, territorio, paisaje, arquitectura, ambiente, estrategias
Recibido: 1 de julio del 2020

Aprobado: 11 de febrero del 2021

doi: https://doi.org/10.26439/limaq2021.n007.5180

This article will explain the strategies used by Chinese architect Kongjian $\mathrm{Yu}$ and his Turenscape team for analyzing and understanding the land as a starting point for the development of his landscape proposals, which challenge the trend of massive use of concrete in China. Returning to the roots of culture, nature and understanding of the land to propose projects that organically manage the variability of nature, especially in its relationship with water, will be related to the incipient development of natural infrastructure in the Peruvian context. Yu's experience manages to link the so-called ecological infrastructure with urban development, whose simile in Peru would be natural infrastructure, which is conceived as the development of natural space associated with the origin of water sources in rural areas, while the implementation of gray infrastructure still prevails in the cities.

natural infrastructure, green infrastructure, water security, land, landscape, architecture, environment, strategies 


\section{INTRODUCCIÓN}

Desde hace una década, a nivel global se escuchan términos como "infraestructura verde", "infraestructura azul", "infraestructura ecológica", y en el Perú, desde el 2018 se cuenta con la normativa que promueve el uso de infraestructura natural como complemento a la infraestructura gris para el manejo del recurso hídrico. Entonces surge una pregunta: ¿qué es la infraestructura natural o verde y por qué es importante?

Según refieren Aguilera, Rodríguez y Gómez (2018) el concepto de infraestructura verde considera las redes que relacionan espacios naturales con espacios construidos o urbanos, y que se desarrollan según los siguientes aspectos o dimensiones: i) multifuncionalidad, ii) conectividad ecológica, iii) conservación, iv) prestación de servicios a la población, y v) multiescalaridad; y concluyen indicando que la infraestructura verde se constituye en una más de las infraestructuras de servicios que aseguran el funcionamiento de los territorios y atienden las necesidades de cualquier sociedad, pudiendo volverse, al mismo tiempo, una oportunidad para repensar las relaciones de los entornos urbanos con el medio. Su importancia radica en la posibilidad de mejorar el medio construido con infraestructura que atiende necesidades propias de un entorno urbano empleando componentes naturales en sus soluciones, y con ello mejorar la calidad de vida de los habitantes y los ecosistemas propios del medio, especialmente cuando se incorporan tecnologías o especies propias. Al respecto, la arquitecta Julia Watson presenta experiencias sobre tecnologías autóctonas que aseguran respuestas sostenibles a problemas como las inundaciones, sobre las cuales las tecnologías contemporáneas asociadas a la ingeniería fracasan en resolver exitosamente, hecho que genera, además, el deterioro del ambiente como parte del proceso (Impakt Festival, 2020).

Tanto Yu (Bengal Institute, 2020) como Watson reconocen el valor de las prácticas y las tecnologías ancestrales. Ambos remarcan que en las culturas ancestrales hay un auténtico conocimiento del manejo del territorio para aprovechar su potencial y prevenir problemas de forma sencilla y muy apropiada a las condiciones y los recursos. $\mathrm{Al}$ darles valor como parte de nuestro conocimiento y luego ponerlas en práctica según nuestras condiciones y tecnología, nos acercamos a un futuro más sostenible.

Este artículo analiza la propuesta de infraestructura ecológica desarrollada por el arquitecto Kongjian Yu debido a la notoriedad que alcanzó desde finales de los noventa con los exitosos resultados obtenidos en la recuperación de medios degradados con proyectos que aún se encuentran vigentes y se han erigido como íconos de un cambio en la tecnología, el diseño y el pensamiento de los ciudadanos y sus gobernantes, gestando una tendencia que cobra cada vez mayor fuerza. El modelo que plantea Yu es coherente al considerar escalas y componentes variados sobre la base de un consciente análisis de base, por lo cual su aplicación es exitosa tanto en China como en otros continentes. 
La conexión de Yu con el territorio se inicia con sus observaciones infantiles de la naturaleza de su entorno rural. Sus intereses se consolidaron al estudiar paisajismo en la Universidad Forestal de Pekín durante los años ochenta y al completar su doctorado en la Universidad de Harvard en 1995. Finalmente, al regresar a China en 1998 funda el Estudio Turenscape y desde ese momento empieza una activa práctica arquitectónica, paisajista y de planificación urbana y territorial en variadas escalas territoriales.

Su trabajo desafió la tendencia de una China que expresaba su desarrollo en el uso masivo del concreto. Él regresó a las raíces de la cultura, la naturaleza y el entendimiento del territorio para plantear proyectos que englobaran de forma orgánica la variabilidad de la naturaleza, especialmente en su relación con el agua. Este experimento, cimentado en sus experiencias adquiridas en Estados Unidos, le permitió definir una estrategia base para el análisis del territorio que priorizó los temas ambientales sobre los estéticos. En su trabajo promueve la planificación y el diseño de una infraestructura ecológica, definida como la estructura de un paisaje sostenible que se constituye a la vez en un límite tangible. Este, a través de propuestas arquitectónicas o paisajistas, impide sobrepasar la capacidad del medio (Yu y Padua, 2006).

En el libro El arte de la supervivencia. Recuperando la arquitectura del paisaje ${ }^{1}$, Yu explica el análisis y metodología empleados en el modelo propuesto para controlar el crecimiento urbano de la ciudad de Taizhou basado en la infraestructura ecológica. Dicho modelo ganó el Premio de Honor ASLA 2005 al Análisis y la Planificación (Yu y Padua, 2006). De acuerdo con el análisis de Kelly Shannon, Yu plantea con sus propuestas que al considerar el arte de la supervivencia en la gestión del territorio y modificar el enfoque de la defensa por la fraternidad con el agua, se logra combatir las inundaciones de manera sostenible. También hace hincapié en las civilizaciones antiguas que tenían métodos ingeniosos para la gestión del agua, abordando simultáneamente pragmatismo, urbanismo y simbolismo. Una ingeniería hidrológica innovadora, el entendimiento de la topografía y los patrones climáticos estacionales tuvieron profundas implicancias en la forma, crecimiento y vitalidad de sus asentamientos. Estas son valiosas lecciones en el legado de los métodos autóctonos de gestión del agua (Shannon, 2013).

En una reciente entrevista realizada en México (Canal Once, 2020), Yu sostiene la importancia del conocimiento consciente del medio para el desarrollo de sus propuestas; y en ese sentido, plantea el estudio de los antecedentes, contexto y cuestiones principales, que en su experiencia están particularmente referidos a temas hídricos, los cuales son enfrentados y resueltos por las ciudades con proyectos de infraestructura que apelan a la resiliencia antes que a la planificación de un desarrollo coherente sin paliativos. Complementa $\mathrm{Yu}$ esta observación indicando que la experiencia del crecimiento acelerado de China hacia el consumismo y la construcción indiscriminada representan una ventaja para los países menos desarrollados, como Perú, donde aún no se ha

1 Su titulo original es The Art of Survival. Recovering Landscape Architecture. 
Figura 1. Expansión territorial en Taizhou

Elaboración propia con información recuperada de Turenscape (2018) llegado al colapso de los recursos naturales, y es posible planificar y diseñar las ciudades y la infraestructura para la mitigación de riesgos en concordancia con el medio y con respeto a la naturaleza (Bengal Institute, 2020).

El proceso metodológico empleado por Turenscape en la definición de los patrones de crecimiento de la ciudad de Taizhou presenta un enfoque integral en el análisis y el entendimiento del territorio, que abarca la región como escenario macro que decanta en la escala local para la aplicación de las propuestas paisajistas. Para logarlo, hace uso de disciplinas como la ecología, la geografía y la hidrología en el estudio físico del territorio y lo complementa con consideraciones culturales propias de cada escala y localidad. En las siguientes figuras se aprecia un resumen de este proceso, según se presenta en el capítulo 2.1 el Patrón de crecimiento de Taizhou, ciudad basada en la infraestructura ecológica (Yu y Padua, 2006).

Antecedentes, contexto y cuestiones principales. El acelerado crecimiento urbano afecta los sistemas hídricos y los humedales, la biodiversidad, los hábitats nativos y la identidad cultural del paisaje.

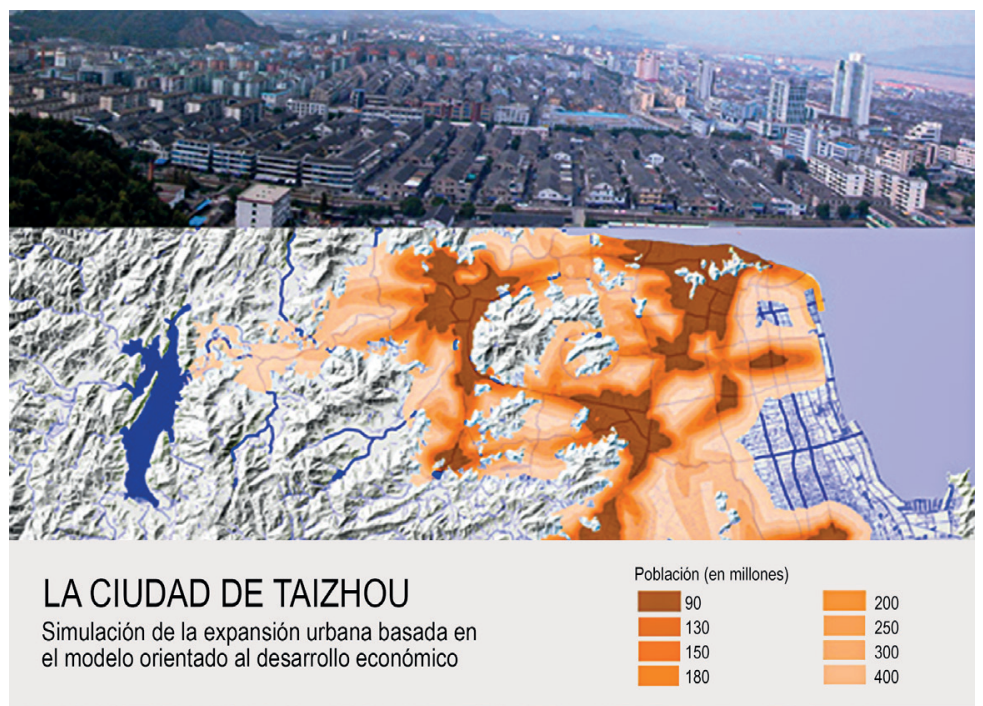

Metas y objetivos del proyecto. Objetivo de los patrones de crecimiento: infraestructura ecológica ${ }^{2}$ que considera el mantenimiento del ecosistema, la protección de los sitios de patrimonio cultural y la creación de oportunidades de recreación.

2 Ecological infraestructure (El según sus siglas en inglés). 
Figura 4. Modelo de seguridad para los procesos naturales

Elaboración propia, con información recuperada de Turenscape (2018)
Definición de la infraestructura ecológica de gran escala. A nivel regional se examinan los patrones críticos, estratégicos en la salvaguarda de los procesos naturales bajo tres niveles: bajo, medio y alto; y se denominan patrones de seguridad.
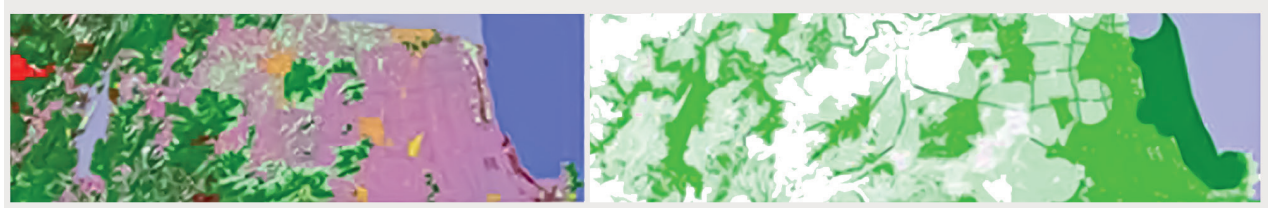

Cobertura del suelo

Análisis de aptitud del hábitat

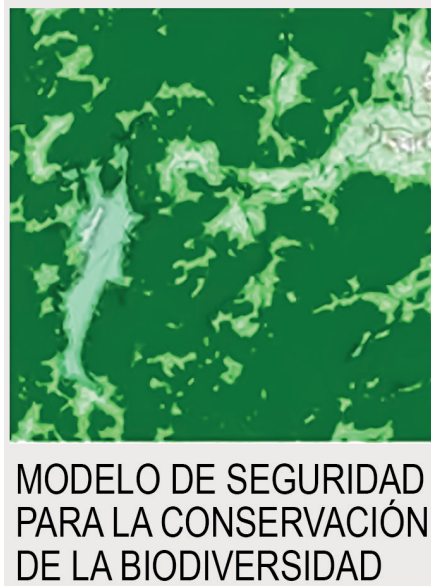

Un comité de planificación selecciona el escenario de calidad media de la IE por ser equilibrado y factible de implementar. Sus miembros son políticos, planificadores expertos, representantes públicos y de la comunidad, promotores inmobiliarios, e inversionistas. 


\section{RED ECOLÓGICA PARA LA DIVERSIDAD PLANIFICADA SEGÚN LOS PRINCIPIOS DEL PAISAJISMO ECOLÓGICO}

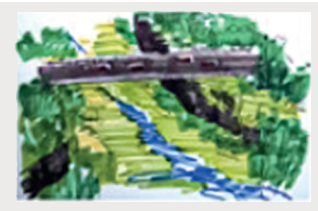

Paso a desnivel para especies y flujos

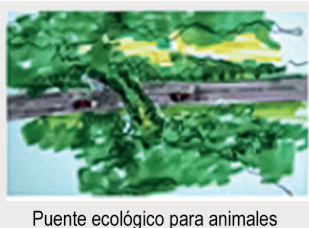

Puente ecológico para animales

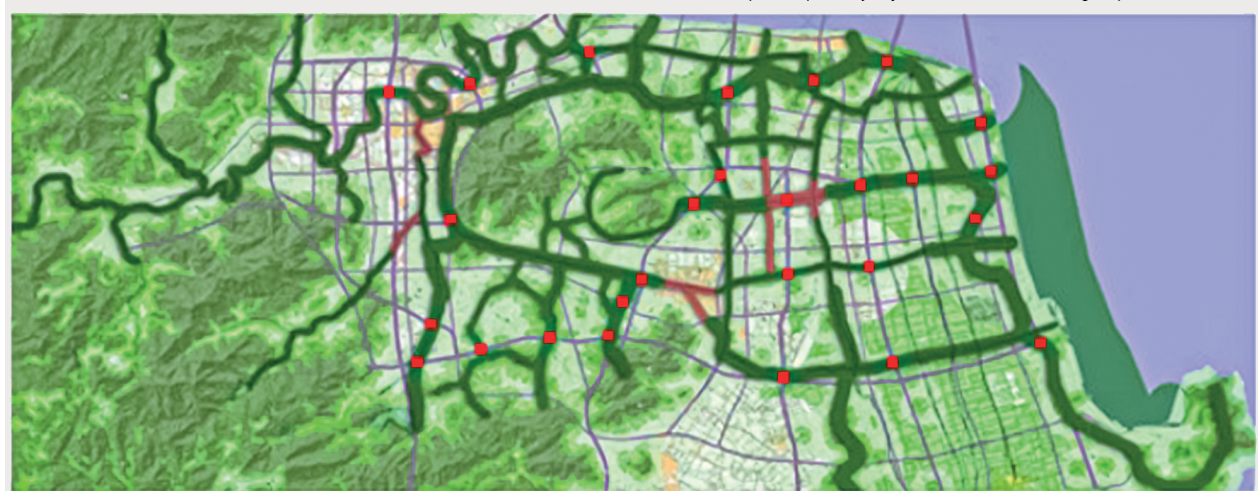

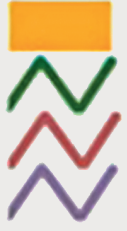

Área construida

Corredores potenciales

Corredores pendientes

Via planificada

- Puntos estratégicos
Fuente - núcleo del hábitat

Área con seguridad baja

Área con seguridad media

Área con seguridad alta
Para proteger la IE regional se definen lazos verdes aprobados por el Congreso Popular en un proceso legislativo que sería el primero en su tipo en China.
Figura 5. Escenario del patrón de crecimiento urbano con base en la infraestructura ecológica regional

Elaboración propia, con información recuperada de Turenscape (2018) 
Figura 6 .

Procedimientos

legales para la

protección de la

infraestructura

ecológica

Elaboración

propia, con

información

recuperada de

Turenscape (2018)
Figura 7.

Definición de la

infraestructura

ecológica de

mediana escala

Elaboración

propia, con

información

recuperada de

Turenscape (2018)
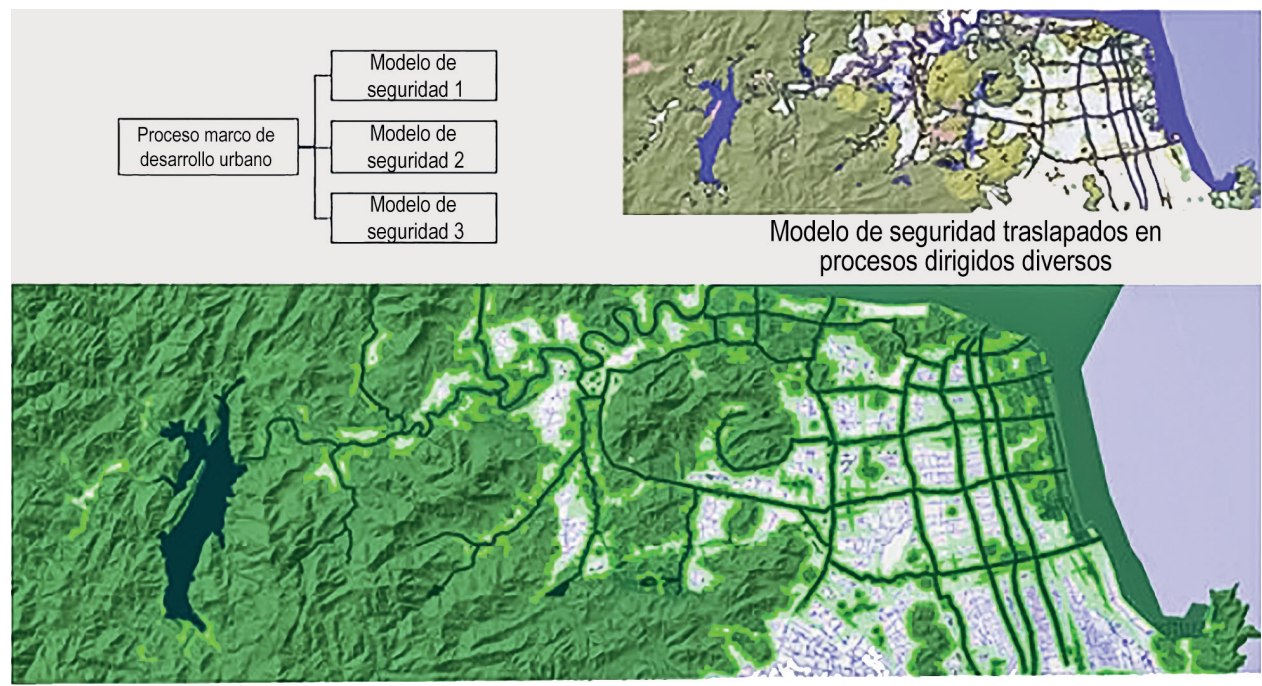

\section{INFRAESTRUCTURA ECOLÓGICA REGIONAL EN TRES NIVELES DE SEGURIDAD}

Nivel de seguridad bajo de IE Nivel de seguridad moderado de IE Nivel de seguridad alto de IE

Involucrando a la comunidad local y sus aportes en el proceso de diseño y propuesta de los lineamientos, se definen corredores verdes: elementos críticos en gestión del agua, conservación de la biodiversidad, protección del patrimonio y recreación.

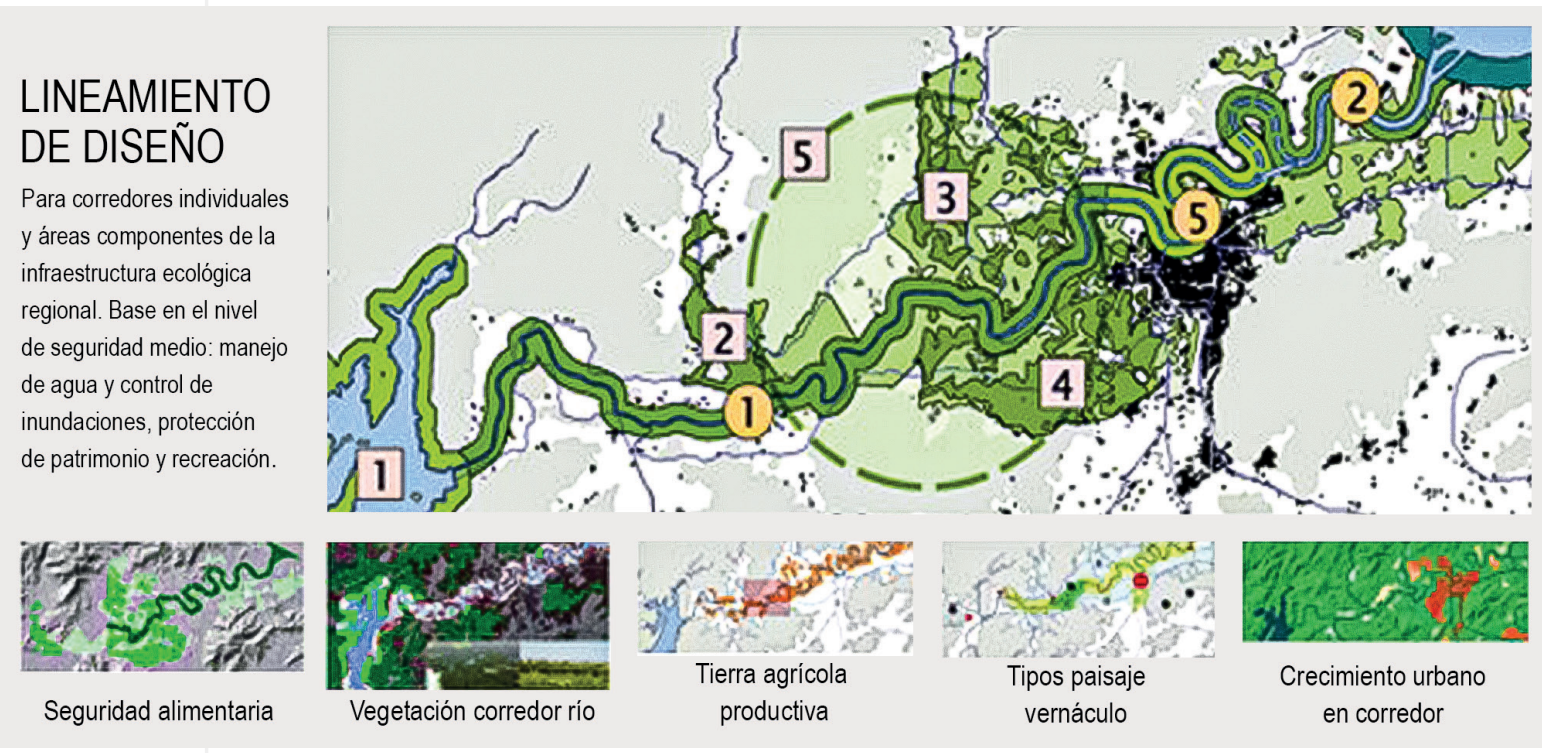


Se presenta a los promotores urbanos, inversionistas y responsables políticos para demostrar su factibilidad en todas las escalas y optimización del potencial del territorio de una manera ecológica y culturalmente sensible.

CLASIFICACIÓN TIPOLÓGICAEN EL CORREDOR DEL RIO BASE PARA LOS LINEAMIENTOS DE DISEÑO
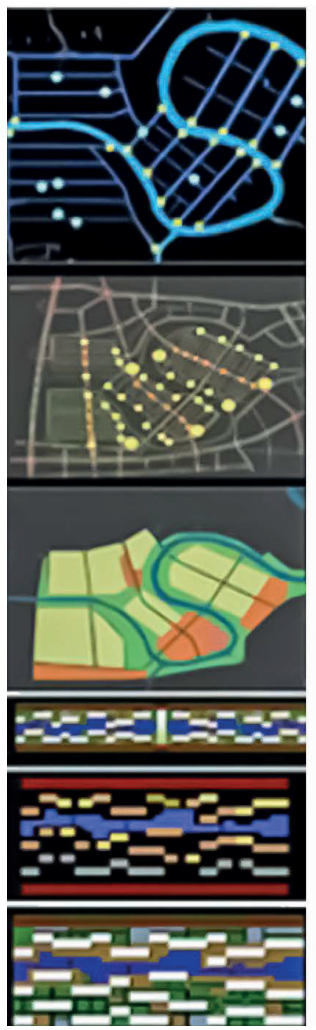

Evitar la inundación reteniendo y redirigiendo el agua, no canalizando y represando. Que un río se convierta en diez arroyos. Que los servicios ecológicos de la infraestructura ecológica penetren en la trama urbana y en las viviendas individuales.

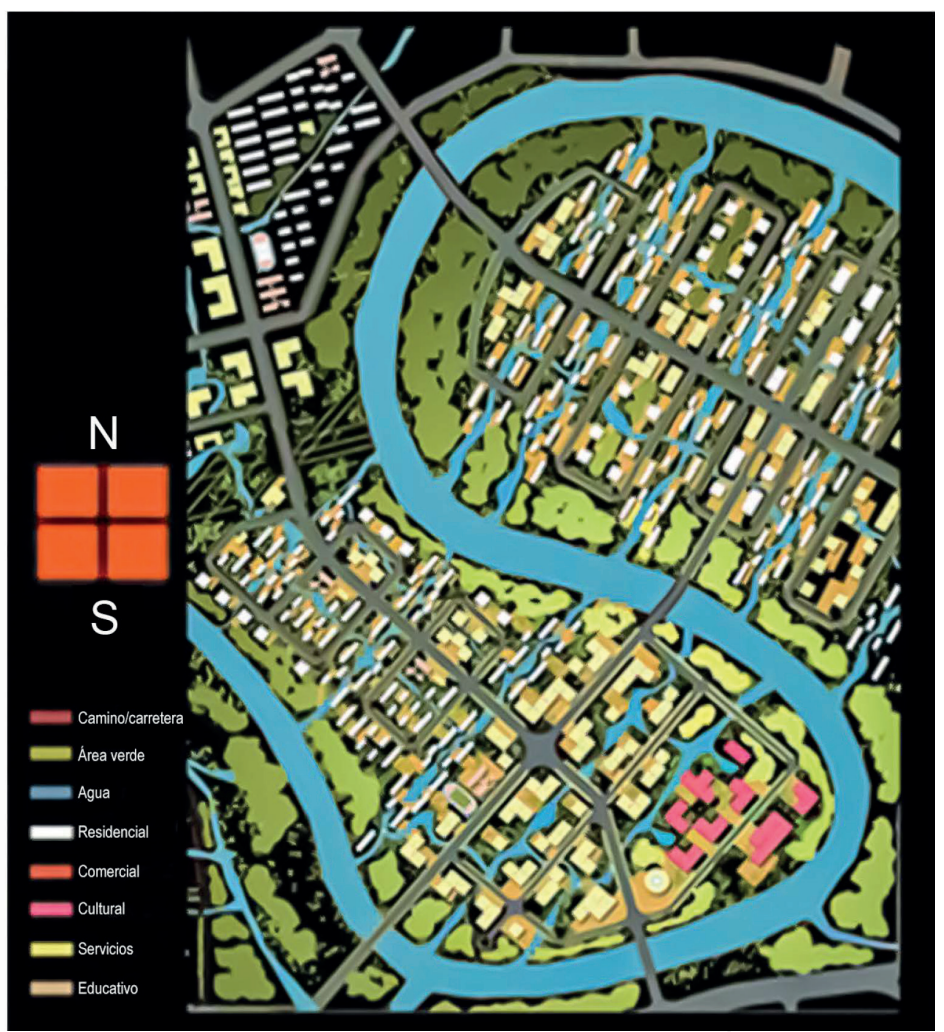

El proceso metodológico antes presentado, a través del cual se desarrolla la propuesta, culmina con la estrategia de implementación que involucra a los principales interlocutores de la localidad, logrando reunir al gobierno con los inversionistas y los habitantes. Este es un tema recurrente en las presentaciones y entrevistas de Yu, la explicación de su estrategia para introducir sus teorías y empezar a implementarlas en China. Él realizó presentaciones ante alcaldes y autoridades locales, que en el contexto chino son quienes deciden el desarrollo de sus localidades, del mismo modo, distribuyó más de veinte mil copias de su libro El camino al paisaje urbano: charlas a los alcaldes ${ }^{3}$ cuando regresó a China para poner en práctica lo aprendido en Estados
Figura 8.

Comprobación de la infraestructura ecológica en la pequeña escala

Elaboración propia, con información recuperada de Yu y Padua (2006)

3 El título original es The Road to Urban Landscape: Talks to Mayors. 
Unidos. Esta estrategia tenía como objetivo principal la educación de las autoridades para que descubrieran las innovaciones que la urbanización occidental estaba experimentando, y a su vez entendieran las consecuencias que la construcción de infraestructura masiva en China en ese momento tendría sobre la naturaleza, tanto por su velocidad como por su agresividad y falta de consideración por el medio. Él mostró a los gobernantes una manera diferente de hacer las cosas y les explicó la belleza que resultaría en el largo plazo al implementar una infraestructura ecológica como solución al desarrollo urbano y a las necesidades del medio, en un momento en el que las intervenciones de ingeniería pesada en China tenían efectos negativos cada vez más evidentes.

Yu manifiesta que el proceso que ha seguido para lograr el cambio no ha sido fácil y que su tenacidad le permitió ser escuchado, atendido y reconocido; también destaca que si bien la educación de las autoridades ha sido crucial, el sostenimiento de esta infraestructura ecológica y su fortalecimiento depende de las generaciones jóvenes. Es por ello que considera que los profesionales actuales deben estar preparados para los retos de la supervivencia. Como decano de la Facultad de Arquitectura y Paisaje de la Universidad de Pekín, prepara profesionales que entiendan la ética ambiental contemporánea tanto como la ciencia y la tecnología, y el interés por negociar la conexión entre el hombre y la naturaleza. También explica que la mejor manera de generar confianza en los gestores es a través de la implementación de modelos y la demostración de la efectividad de las teorías. Como consecuencia de ello, se forman valores y cultura en los usuarios, sobre la sostenibilidad y los paisajes ecológicos y sostenibles, y se genera impacto en los medios de comunicación, que a su vez se convierten en difusores de estas nuevas prácticas y este nuevo conocimiento. A partir de su interpretación personal de la difusión en redes de sus proyectos hecha por los usuarios de sus proyectos, Yu plantea que incluso las redes sociales sirven como medios generadores de educación.

\section{EN EL PERÚ}

En el contexto peruano, las propuestas de infraestructura verde se plantean en complemento a soluciones de infraestructura gris, compuestas por complejos proyectos de ingeniería, que repiten expedientes técnicos típicos sin incluir una evaluación sobre la eficacia de los resultados obtenidos. Poco se ha escrito o legislado sobre la necesidad de incluir a la infraestructura natural o a los sistemas urbanos sostenibles (Abellán, 2013) como componente crítico del desarrollo urbano en relación con la conservación ambiental y la mejora de la calidad de vida de la comunidad.

En el 2015 se definieron los Lineamientos de Política de Inversión Pública en materia de Diversidad Biológica y Servicios Ecosistémicos 2015-2021, a través de la Resolución Ministerial n. ${ }^{\circ}$ 199-2015-MINAM, que sentó las bases legales para que se considere la conservación, uso sostenible de la diversidad biológica y los servicios ecosistémicos en las inversiones públicas a nivel nacional. En esta línea, con la Resolución Ministerial n. ${ }^{\circ}$ 178-2019-MINAM, se plantean los 
Lineamientos para la Formulación de Proyectos de Inversión en las Tipologías de Ecosistemas, Especies y Apoyo al Uso Sostenible de la Biodiversidad, que involucra al Ministerio de Economía y Finanzas en la puesta en práctica a través de las inversiones públicas en las acciones ambientales. Esto con el objetivo de cerrar las brechas relacionadas con la degradación de ecosistemas naturales, y que incluye a la infraestructura natural, definida como la red de espacios naturales que conservan los valores y funciones de los ecosistemas, proveyendo servicios ecosistémicos (MINAM, 2019). Este esfuerzo también se está complementando con el empuje del Ministerio del Ambiente por establecer una cartera de proyectos de inversión en la tipología de infraestructura natural que permita identificar proyectos de interés mutuo entre el sector público y privado. La meta es asegurar los beneficios económicos, sociales y ambientales a la sociedad, mediante la ejecución de proyectos que apunten hacia la recuperación y conservación de este tipo de infraestructura existente en el país (Plataforma Digital Única del Estado Peruano, 2020).

Por otra parte, a partir de la creación de la Autoridad para la Reconstrucción con Cambios después del fenómeno de El Niño de 1997, se priorizó la intervención e inversión pública en siete departamentos de la costa, desde Tumbes hasta Ica, planteando planes integrales para el control de inundaciones y movimientos en masa como soluciones integrales para la prevención de desastres. Entre sus planteamientos figuran los siguientes: (a) defensas ribereñas para reducir los riesgos en un $60 \%$, (b) presas y reservorios para reducir los riesgos en un $35 \%$, y (c) infraestructura natural, que abarcaría un $5 \%$ de todas las inversiones. Esta última complementaría la infraestructura gris en intervenciones usualmente planteadas para infraestructura de gran escala, la cual se orientaría a la cosecha de agua en las partes altas de las cuencas, forestación o similares (Aucasime, 2020) , $^{4}$ sin acercarse a las intervenciones urbanas, en las que, con montos de inversión menores, se puede lograr soluciones más eficientes y mejoras notables en la calidad de vida urbana.

4 Como se puede ver en Montenegro (2018), que muestra imágenes de las soluciones integrales en ríos y quebradas de la Reconstrucción con Cambios. Véase el siguiente QR:

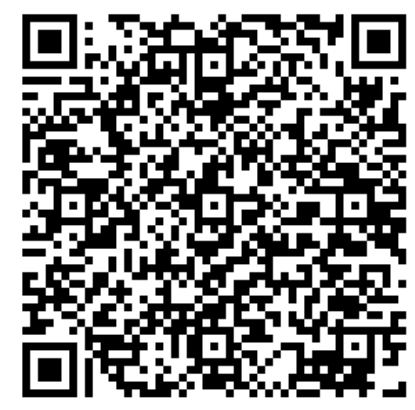




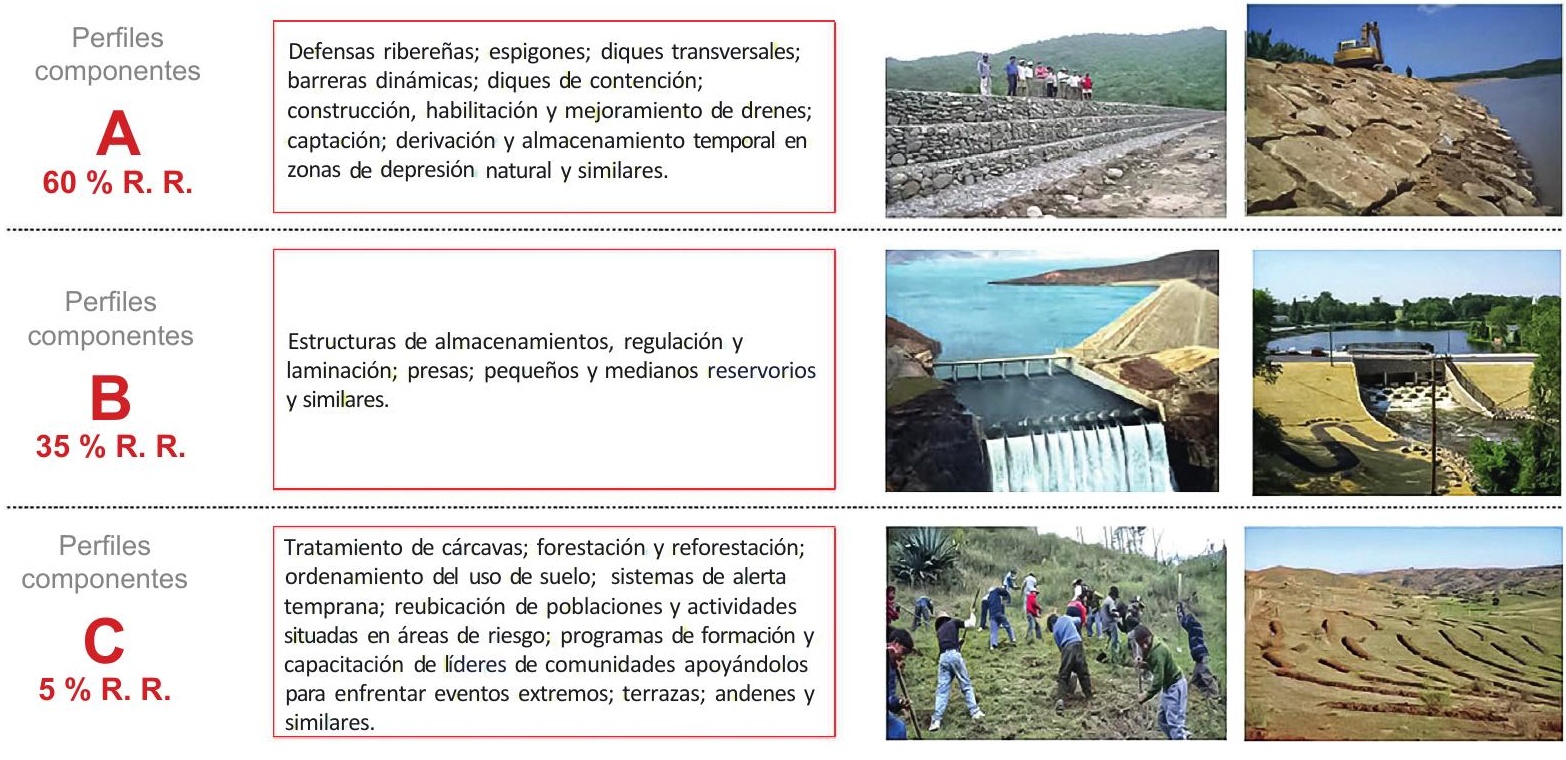

Figura 9. En paralelo, y como parte de las iniciativas nacionales en planificación urbana, a finales del 2018 se inició el proceso de planificación más ambicioso en la historia del urbanismo nacional, la contratación pública especial de consultorías para la elaboración de planes de acondicionamiento territorial (PAT), planes de desarrollo metropolitano (PDM) y planes de desarrollo urbano 


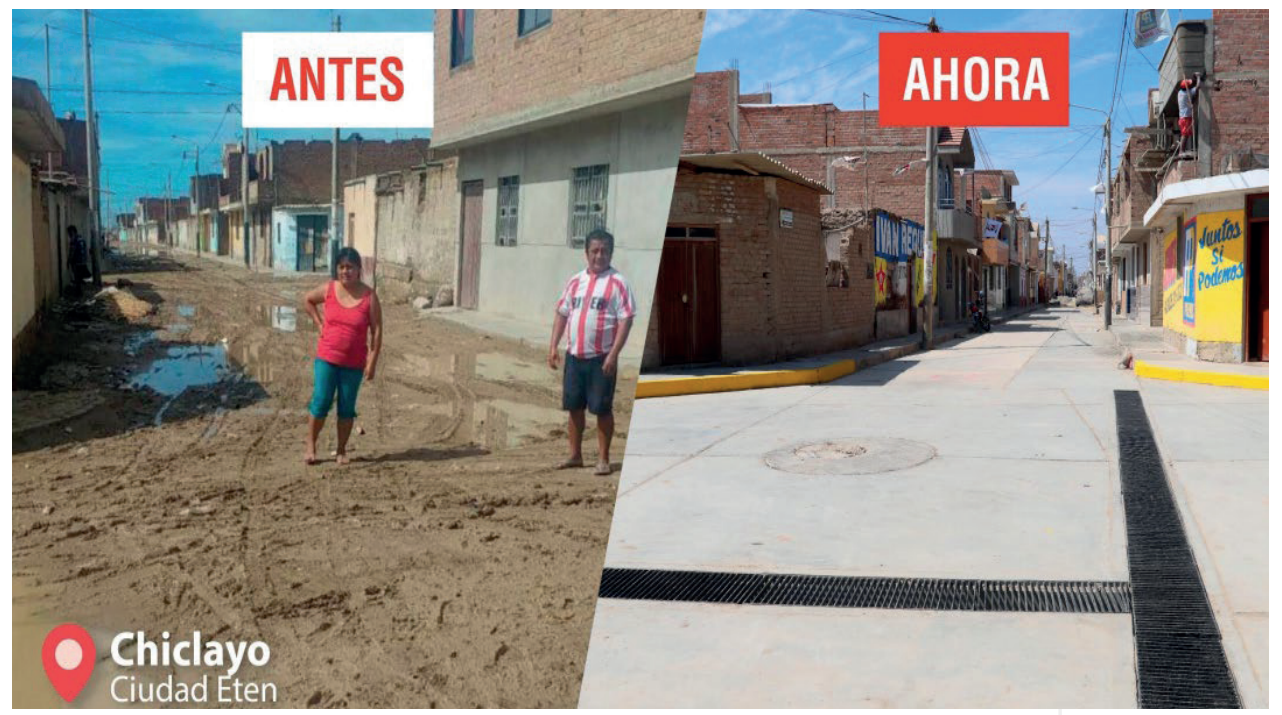

ambiental se desarrolla en los siguientes aspectos: (1) como parte del diagnóstico, en el Análisis de la vulnerabilidad ambiental de riesgos, y (2) como un componente de los objetivos de priorización de los planes en la delimitación del ámbito de intervención: "establecer mecanismos de evaluación y monitoreo ambiental en las ciudades". Este análisis, que se realiza durante la etapa de diagnóstico, no tiene una secuencia clara en la etapa propositiva, por lo que el resultado final de los planes mantiene un enfoque de solución rígida y eficiente desde la ingeniería, que actualmente deja de lado la inclusión de infraestructura natural, en parte debido a que desde el análisis inicial carece de un entendimiento más amplio de lo ambiental como un territorio vivo, aún dentro de las ciudades.

Además, la normativa sobre planificación urbana deja pendiente la adaptación y consideración de las ciudades como espacios relacionados a un entorno natural, sobre todo teniendo en cuenta que muchas de las capitales distritales se encuentran aún en transición hacia lo urbano, pues mantienen muchas características de un medio rural, sea por cantidad de población, densidad e incluso desarrollo económico, que en muchos casos es principalmente agrícola.

De acuerdo con el modelo de Turenscape para Taizhou, se aprecia que es necesaria una mayor articulación entre ministerios y una mayor apertura por parte del Ministerio de Vivienda para incorporar como parte de la normativa, y de manera más inmediata en los planes que actualmente se están elaborando, los lineamientos técnicos que ya ha definido y gestionado el Ministerio del Ambiente. Es importante también que los arquitectos tomen mayor interés por el ambiente y que se reconozca que las intervenciones menos tangibles pueden ser las de mayor importancia para generar el cambio hacia lo sostenible y a la misma vez mitigar riesgos que originan grandes pérdidas económicas y humanas.
Figura 10.

Soluciones de drenaje urbano. Teoría versus práctica

\section{Elaboración} propia, con información recuperada de Montenegro (2018) 
Según indican Cobo y Piñeiros (2020) sobre la base del estudio de la ONU en relación al manejo del agua con soluciones naturales (WWAP, 2018),

la infraestructura verde urbana se utiliza cada vez más para gestionar y reducir la contaminación provocada por la escorrentía urbana. Los ejemplos incluyen muros verdes, jardines en los techos y cuencas de infiltración o drenaje cubiertas de vegetación para contribuir al tratamiento de las aguas residuales y reducir la escorrentía de las aguas pluviales. También se utilizan los humedales en entornos urbanos para mitigar el impacto de la escorrentía de las aguas pluviales y las aguas residuales contaminadas. Los humedales, tanto naturales como construidos, también contribuyen a la biodegradación o inmovilización de toda una gama de contaminantes emergentes, incluidos ciertos productos farmacéuticos y a menudo funcionan mejor que las soluciones grises. Para ciertos productos químicos pueden ser la única solución. (p. 4)

$\mathrm{Al}$ respecto, existen ejemplos notables, como en la localidad de VitoriaGasteiz, al norte de España, donde son comunes como intervenciones en las vías los depósitos de infiltración y los drenajes sostenibles; en las edificaciones, los jardines de lluvia y los sistemas de recojo de agua pluvial; en la estructura urbana, los jardines verticales y techos verdes; y de manera dispersa en la ciudad y su entorno, las áreas verdes de pequeño tamaño o microespacios de biodiversidad que permiten la recuperación de ecosistemas fluviales urbanos, así como la generación de hábitats para insectos, pequeñas aves y polinizadores (CEA, 2014). Estas potenciales intervenciones de infraestructura verde, que puede aplicarse desde la escala urbana a la regional, también han sido claramente propuestas para el corredor ribereño del río Mapocho en Santiago de Chile (Vásquez, 2016), que en la siguiente tabla presenta claramente las posibilidades de incluir la infraestructura natural como parte de la planificación y diseño urbano.

Tabla 1

Componentes del paisaje con potencial de

infraestructura verde

Elaboración propia, con información recuperada de Vásquez (2016)

\begin{tabular}{lll}
\hline \multicolumn{1}{c}{ ESCALA DE BARRIO } & \multicolumn{1}{c}{ ESCALA DE CIUDAD } & \multicolumn{1}{c}{ ESCALA REGIONAL } \\
\hline Calles arboladas & Ríos y llanuras de inundación & Áreas silvestres protegidas \\
Techos y paredes verdes & Parques intercomunales & Parques nacionales \\
Plazas de barrio & Canales urbanos & Bordes costeros y playas \\
Jardines privados & Lagunas & Senderos estratégicos y de \\
Espacios abiertos & Bosques urbanos & larga distancia \\
institucionales & Parques naturales & Bosques \\
Estanques y arroyos & Frentes de agua continuos & Fajas de resguardo en líneas \\
Derechos de paso de & Plazas municipales & de alta tensión \\
caminos peatonales y & Cerros & Red de carreteras y \\
ciclorrutas & Grandes espacios recreativos & ferrocarriles \\
Cementerios & Esteros & Cinturón verde designado \\
Pistas deportivas & Terrenos abandonados & Tierras agrícolas \\
Zanjas de inundación & Bosques comunitarios & Ríos y lagunas de inundación \\
Pequeños bosques & Sitios mineros en abandono & Canales \\
Áreas de juego & Tierras agrícolas & Campo abierto \\
Quebradas & Vertederos & Cordones montañosos \\
Patios de escuelas & & Territorio de propiedad común \\
Huertos & & Acueductos y gaseoductos \\
Terrenos abandonados & & Fallas geológicas lagos \\
\hline
\end{tabular}




\section{GESTIÓN DEL AGUA}

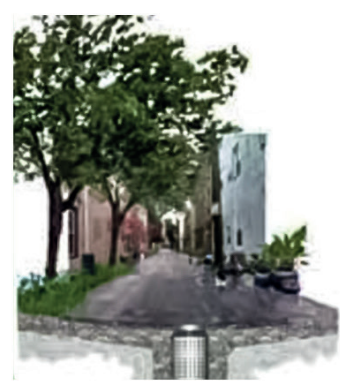

Transformación de los sistemas convencionales de tratamiento de aguas pluviales en sistemas urbanos de drenaje sostenible reduciendo la carga de aguas pluviales en el alcantarillado. Proyecto Green City, Clean Waters, Filadelfia.

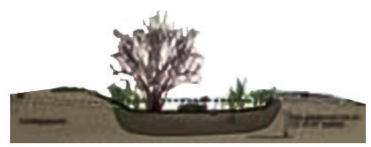

Jardines de lluvia actúan como filtro de las aguas de escorrentia reduciendo los contaminantes que entran a los sistemas de saneamiento. Washington.

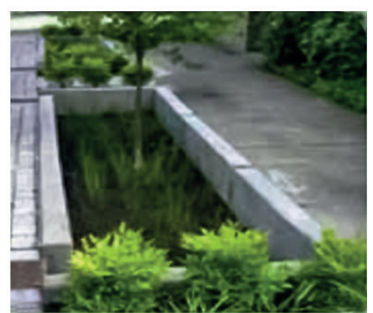

Depósitos de infiltración que actúan come zonas de embalse superficial donde se almacena el agua hasta que se produce la infiltración. Portland.

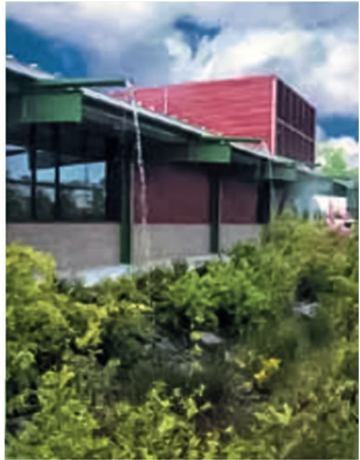

Sistemas de recogida de aguas pluviales Portland.
DISEÑO Y GESTIÓN DEL VERDE URBANO

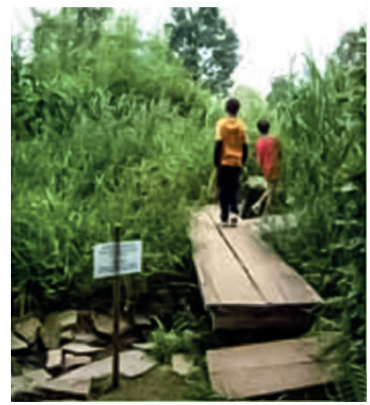

Recuperación de los ecosistemas fluviales urbanos con diseños que favorecen el acercamiento al agua. Chaumont-Sur-Loire.

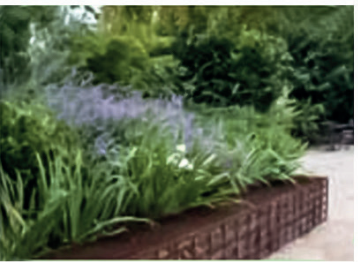

Integración de diseños urbanos funcionales y generación de hábitats para insectos, pequeñas aves y polinizadores.

Chaumont-Sur-Loire

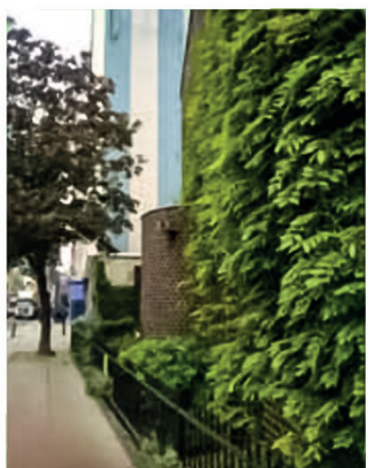

Jardines verticales que incrementan la superficie de espacio verde y el indice biótico del suelo. Londres.

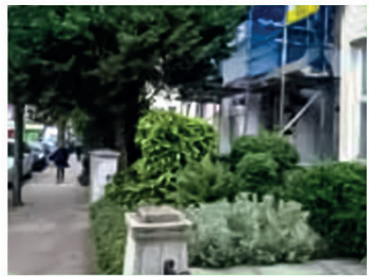

Conservación de microespacios de biodiversidad en los procesos de renovación urbana. Londres.

\section{CONCLUSIÓN}

$\mathrm{Al}$ conocer las tendencias en el pensamiento conservacionista, las experiencias exitosas de proyectos en diversas escalas que relacionan la naturaleza con el desarrollo urbano y la normativa que ampara y asegura la implementación, y también el financiamiento de estas innovaciones, es importante llamar al compromiso de los profesionales involucrados, arquitectos e ingenieros, a cerrar el círculo de la planificación y el diseño urbano articulado a la infraestructura natural, considerando para ello:

- Incluir en los proyectos las herramientas técnicas y legales que el Estado peruano está formulando, exigiendo su aplicación aprovechando los mecanismos de financiamiento mediante inversiones públicas. 
- Hacer prevalecer el análisis territorial integral por sobre el políticoadministrativo, empleando, por ejemplo, el enfoque de cuencas, de modo que se considere la modelación de procesos integrales y dinámicos. Para ello será importante fortalecer las capacidades de negociación entre autoridades involucradas en procesos comunes.

- Fortalecer las políticas de capacitación a autoridades, funcionarios y profesionales consultores o proyectistas para asegurar la aplicación integrada de la infraestructura natural en todos los niveles.

- Analizar los componentes ambientales del territorio de forma dinámica, tanto en sus dinámicas como en su temporalidad, y formalizar la realización de estudios cualitativos que cubran y complementen las deficiencias estadísticas con las cuales se desarrollan los estudios actualmente; de modo que las propuestas se sostengan y se guíen hacia la mejora de la calidad del medio y de sus habitantes.

- Masificar el uso de herramientas de análisis territorial, como el Sistema de Información Geográfica (SIG) que actualmente emplean algunas municipalidades y equipos profesionales planificadores sin aprovechar los recursos que suponen para la planificación.

- Comprometer a los actores relevantes en cada escala, sea la cooperación internacional, las instituciones y empresas privadas, las asociaciones públicas, las instituciones educativas, las asociaciones de base, y los emprendimientos individuales; a sumarse de manera conjunta a la conservación del ambiente.

El enfoque del trabajo de Yu es acertado desde la técnica y la tecnología, pues sus proyectos han demostrado que la regeneración de la naturaleza y de la cultura local es posible con una intervención pensada y estructurada. También ha sido acertada su gestión, pues al educar a los gobernantes ha logrado la aceptación y sobre todo el entendimiento de sus ideas, desde los gestores hasta los usuarios, pasando por los medios de comunicación nacionales e internacionales; y sobre todo, su trabajo es acertado porque al originarse en lo propio del territorio y de la cultura ha generado el retorno de la naturaleza y sus ecosistemas, de la sostenibilidad basada en los saberes populares y ha fortalecido la apropiación de los conceptos empleados en la práctica paisajista, calando en el orgullo de sus usuarios.

Por estas razones toma valor la experiencia china, que ha reconsiderado su crecimiento desenfrenado para escuchar a un soñador y permitirle construir con delicadeza espacios con calidad dentro de la ciudad. En el Perú tenemos una gran naturaleza y una maravillosa cultura como base, es hora de que continuemos con esta nueva línea de acción.

En concordancia con la opinión de Pickett, Cadenasso y McGrath (2013), los arquitectos, urbanistas, planificadores y paisajistas están actualmente 
imaginando las ciudades en su relación con el agua. Proyectos que reúnen procesos naturales y diseñados están fortaleciendo las lógicas propias de cada localidad y se están convirtiendo en la base de nuevas formas urbanas y regionales. Hoy el enfoque de ingeniería blanda es promovido como una forma de relacionarse con las fuerzas de la naturaleza con el fin de reducir o mitigar los impactos de los desastres naturales, mientras el desarrollo ajustado de las ciudades debe ser guiado por nuevas interacciones del paisaje, la infraestructura y la urbanización. No podemos quedarnos rezagados.

\section{REFERENCIAS}

Abellán, A. (2013). ¿Qué son los SUDS? SuD Sostenible. Recuperado de http:// sudsostenible.com/que-son-los-sistemas-urbanos-de-drenaje-sostenible/

Aguilera, F., Rodríguez, V. y Gómez, M. (2018). Definición de infraestructuras verdes: una propuesta metodológica integrada mediante análisis especial. Documents D'Anàlisi Geogràfica, 64(2), 313-337. doi: https://doi. org/10.5565/rev/dag.419

Aucasime, A. (2020). Webinar: Reconstrucción con Cambios: Soluciones sostenibles con infraestructura natural [Archivo de video]. Recuperado de https://www. youtube.com/watch?v=NHtveXUwJ9U\&t=1378s

Bengal Institute. (2020). Conversation Between Kongjian Yu and Kazi Khaleed Ashraf-Bl Between 01 [Archivo de video]. Recuperado de https://www. youtube.com/watch?v=iyCgWcMiwLw

Calaza Martínez, P. (2016). Trees in Urban Ecosystem: Connection Between New Urbanism, Society and Rational Risk Management. Ingeniería y Universidad, 20(1), 155-173. doi: http://dx.doi.org/10.11144/Javeriana.iyu 20-1.tuec

Canal Once. (2020). Espiral-Entrevista con Kongjian Yu [Archivo de video]. Recuperado de https://www.youtube.com/watch?v=qixY6SOTfng

CEA. (2014). La infraestructura verde urbana de Vitoria-Gasteiz. Documento de propuesta. Centro de Estudios Ambientales. Ayuntamiento de VitoriaGasteiz. Vitoria-Gasteiz.org Recuperado de https://www.vitoria-gasteiz.org/ wb021/http/contenidosEstaticos/adjuntos/eu/32/95/53295.pdf

Chaudhry, R. (2005). The Growth Pattern of Taizhou City Based on Ecological Infrastructure. Turenscape. Recuperado de https://www.turenscape.com/ en/news/detail/121.html

Cobo, E. y Piñeiros L. (2020). Infraestructura Natural: Oportunidades para optimizar la gestión de sistemas hídricos. Quito: UICN.

COUS UNAM. Coordinación Universitaria para la Sostenibilidad. Universidad Nacional Autónoma de México. (2019). Kongjian Yu. Teoría y práctica de patrones estratégicos de seguridad ecológica [Archivo de video]. Recuperado de https://www.youtube.com/watch?v=WkKyYQGGfwQ\&list=PL-yZGFjyHUgf T7kJsQrqnuyrkM5ymzv26\&index=5\&t=2567s 
Cueva, S. (2020). Estudios de Preinversión Proyectos de Soluciones Integrales. PCM-RCC. Recuperado de https://www.rcc.gob.pe/2020/wp-content/ uploads/2020/09/Presentacion-Estudios-RoadShow-G2G-DAI-VF.pdf

De Meulder, B. y Shannon, K. (2011). Ecological Structures to Guide Urbanization: Landscape Urbanization in Vietnam. Journal of Landscape Architecture, (31), pp. 38-45.

Decreto Supremo n. ${ }^{\circ}$ 022-2016-VIVIENDA, por el cual se aprueba el Reglamento de Acondicionamiento Territorial y Desarrollo Urbano Sostenible. (2016). Recuperado de https://busquedas.elperuano.pe/normaslegales/decreto-su premo-que-aprueba-el-reglamento-de-acondicionamien-decretosupremo-n-022-2016-vivienda-1466636-3/\#: :text=El\%20presente\%20 Reglamento\%20tiene\%20por,1.

Fariña, J. (27 de junio del 2012). Infraestructura Verde Urbana [mensaje en un blog]. Recuperado de https://elblogdefarina.blogspot.com/2012/06/infraes tructura-verde-urbana.html

Forest Trends América Latina. (2020). Webinar: Reconstrucción con Cambios:Soluciones sostenibles con infraestructura natural. [Archivo de video]. Recuperado de https://www.youtube.com/watch?v=NHtveXUwJ9U\&list=PL-yZGFjyHUgc4 gCCy_4SpbRDxxZb0h9J3\&index $=17 \& t=3037 \mathrm{~s}$

García Sánchez, F. (2019). Planeamiento urbanístico y cambio climático: la infraestructura verde como estrategia de adaptación. Cuadernos de Investigación Urbanística, (122), 2019. doi:10.20868/ciur.2019.122.3870

Harvard Graduate School of Design. (2008). Kongjian Yu, "The Art of Survival: Recovering Landscape Architecture" [Archivo de video]. Recuperado de https://www.youtube.com/watch?v=JH96L4JxMyA\&list=PL-yZGFjyHUgfT7 kJsQrqnuyrkM5ymzv26\&index $=4 \& t=0$ s

Impakt Festival. (2020). Douglas Rushkoff in Conversation with Julia Watson @ IMPAKT Festival 2020 [Archivo de video]. Recuperado de https://www. youtube.com/watch?v=tu1 IEjH6IVg\&t=1233s

Ministerio de Economía y Finanzas. (2015). Lineamientos para la Formulación de Proyectos de Inversión Pública en Diversidad Biológica y Servicios Ecosistémicos. Lima: DGIP-MEF.

Ministerio del Ambiente. (2018). Inversión pública en infraestructura natural. MINAM. Recuperado de http://www.minam.gob.pe/economia-y-financiamiento-am biental/inversion-publica-en-servicios-ecosistemicos/

Ministerio de Vivienda, Construcción y Saneamiento. (2018). Manual para la elaboración de Planes de Desarrollo Urbano y Planes de Desarrollo Metropolitano en el marco de la Reconstrucción con Cambios. Lima: MVCS.

Ministerio de Vivienda, Construcción y Saneamiento. (2018). Contratación de Servicios de Consultoría en General. Recuperado de https://ww3.vivienda. gob.pe/ProcesosCE/ 
Montenegro, J. L. (2018). Autoridad para la Reconstrucción con Cambios. Soluciones integrales. Intervenciones de construcción considerados en el Plan Integral de la Reconstrucción con Cambios. Recuperado de https://www.senace. gob.pe/wp-content/uploads/2019/02/Evento-IGAPRO-Intervenciones-deConstruccion-considerados-en-el-Plan-Integral-de-la-Reconstruccion-conCambios.pdf

Montenegro, J. L. (2019). Autoridad para la Reconstrucción con Cambios. Soluciones Integrales. Intervenciones de Construcción considerados en el Plan Integral de la Reconstrucción con Cambios. Recuperado de https://www.senace. gob.pe/wp-content/uploads/2019/03/Tema-3-Soluciones-Integrales-en-lasIntervenciones-de-Construccion.pdf

Ndubisi, F. O. (2014). The Ecological Design and Planning Read. Washington, D. C.: Island/Center for Resource Economics.

Pickett, S., Cadenasso, M, y McGrath, B. (2013). Eco-Engineering for Water: From Soft to Hard and Back. In Resilience in Ecology and Urban Design: Linking Theory and Practice for Sustainable Cities, vol. 3. Londres: Springer.

Plataforma Digital Única del Estado Peruano. (2020). Inversión en infraestructura natural es clave para garantizar la seguridad hídrica del país. Recuperado de https://www.gob.pe/institucion/minam/noticias/81425-inversion-en-infraes tructura-natural-es-clave-para-garantizar-la-seguridad-hidrica-del-pais

Quispe, E. (2018). Reconstrucción e institucionalidad con visión de cambio. PCM-RCC. Recuperado de http://www.cip.org.pe/publicaciones/isdrm/presentaciones ISDRM2018/MSc-Edgar-Quispe.pdf

Reconstrucción con Cambios. (2019). Infraestructuras de prevención. Ríos y quebradas a intervenir por la Autoridad para la Reconstrucción con Cambios. RCC-PCM. Recuperado de https://www.rcc.gob.pe/2020/main-home/planintegral/prevencion/

Resolución Ministerial n. ${ }^{\circ}$ 178-2019-MINAM. Por la cual se establecen los Lineamientos para la formulación de proyectos de inversión en las tipologías de ecosistemas, especies y apoyo al uso sostenible de la biodiversidad. (7 junio de 2019). Recuperado de https://cdn.www.gob.pe/uploads/document/ file/319848/RM_N_178-2019.pdf

Resolución Ministerial n. ${ }^{\circ}$ 199-2015-MINAM. Por la cual se establecen los Lineamientos de política de inversión pública en materia de diversidad biológica y servicios ecosistémicos 2015-2021. (11 de agosto del 2015).

Román, F., Estévez, G., Aste, N. y Moles, A. (2020). HIRO, Herramienta de identificación rápida de oportunidades para la infraestructura natural en la gestión del riesgo de desastres. Guía Metodológica. Recuperado de https:// www.forest-trends.org/wp-content/uploads/2020/04/GUIA-HIRO-GRD-1. pdf

Saunders, W. S. (2013). Designed Ecologies: The Landscape Architecture of Kongjian Yu. Basel: Birkhäuser. 
SPDA Actualidad Ambiental. (2018). Infraestructura natural: Aprovechar recursos de la naturaleza y conservarla a la vez. Recuperado de https://www. actualidadambiental.pe/infraestructura-verde-aprovechar-recursos-de-lanaturaleza-y-conservarla-la-vezl

Turenscape. (2018). Taizhou Ecological Infrastructure. The Growth Pattern of Taizhou City Based on Ecological Infrastructure-The Negative Approach Physical Urban Planning. City Planning Review, 2005(9), 76-80. Recuperado de https://www.turenscape.com/news/detail/1185.html

Vásquez, A. (2016). Infraestructura verde, servicios ecosistémicos y sus aportes para enfrentar el cambio climático en ciudades: el caso del corredor ribereño del río Mapocho en Santiago de Chile. Revista de Geografía Norte Grande, (63), 63-86. Recuperado de https://scielo.conicyt.cl/pdf/rgeong/n63/art05.pdf

Wang, S., Ke Y., Donghai Y., Kongjian Y., y Yijing S. (2019). Temporal-Spatial Changes about the Landscape Pattern of Water System and Their Relationship with Food and Energy in a Mega City in China. Ecological Modelling, 401, 75-84.

WWAP (Programa Mundial de las Naciones Unidas de Evaluación de los Recursos Hídricos)/ONU-Agua. (2018). Informe Mundial de las Naciones Unidas sobre el Desarrollo de los Recursos Hídricos 2018: Soluciones basadas en la naturaleza para la gestión del agua. París, UNESCO. Recuperado de https://unesdoc.unesco.org/in/documentViewer.xhtml?v=2.1.196\&id=p:: usmarcdef_0000261494\&file=/in/rest/annotationSVC/Download WatermarkedAttachment/attach_import_7e99b75b-efad-4db2-8cc3da0007893705\%3F_\%3D261494spa.pdf\&locale=es\&multi=true\&ark=/ ark:/48223/pf0000261494/PDF/261494spa.pdf\#\%5B\%7B\%22num\%22\%3A $82 \% 2$ C $\% 22$ gen $\% 22 \% 3$ A0\%7D \%2C \% 7B $\% 22$ name $\% 22 \% 3$ A $\% 22 X Y Z \% 22 \%$ $7 \mathrm{D} \% 2 \mathrm{C}-1 \% 2 \mathrm{C} 842 \% 2 \mathrm{C} 0 \% 5 \mathrm{D}$

Yu, K. y Padua, M.G. (2006). The Art of Survival. Recovering Landscape Architecture. Mulgrave y Victoria: The Images Publishing Group Pty Ltd.

Zeunert, J. (s. f.). Interview: Knogjian Yu. Bloombury: Landscape Architecture and Environmental Sustainability. Recuperado de https://www.bloomsbury.com /cw/landscape-architecture-and-environmental-sustainability/interviews/ kongjian-yu/\#: :text=Professor\%20Kongjian\%20Yu\%20is\%20a,Planning $\% 20$ and $\% 20$ Design $\% 2 \mathrm{C} \% 20 \mathrm{Harvard} \% 20$ University.

Zhao, J., Kongjian Y., y Dihua L. (2014). Spatial Characteristics of Local Floods in Pekin Urban Area. Urban Water Journal 11.7, 557-72. 